\title{
Epigenetic instability is rare in fibrolamellar carcinomas but common in viral-associated hepatocellular carcinomas
}

\author{
Perumal Vivekanandan and Michael Torbenson \\ Department of Pathology, The Johns Hopkins University School of Medicine, Baltimore, MD, USA
}

\begin{abstract}
Fibrolamellar carcinomas have a unique predilection for younger individuals and arise in livers without recognizable liver disease. In contrast to typical hepatocellular carcinomas, fibrolamellar carcinomas show few chromosomal changes and lack mutation in key genes such as TP53 and CTNNB1. Epigenetic instability, manifesting as methylation of important tumor suppressor gene promoters, has not been investigated in fibrolamellar carcinomas. Thus, the methylation status of 11 tumor suppressor gene promoters was investigated using methylation-specific PCR: RASSF1, CDH1, CDKN2B, HPP1, CDKN2A, GSTP1, P16, RARA, FLJ13081, SOCS1, and TP53. Nine fibrolamellar carcinomas were studied including primary tumors $(N=5)$ and metastatic deposits $(N=4)$ along with control groups of typical hepatocellular carcinoma arising in livers with $(N=21)$ and without cirrhosis $(N=10)$. In fibrolamellar carcinomas, RASSF1A and CDH1 (e-cadherin) were the most commonly methylated genes with $\mathbf{8 0 - 1 0 0 \%}$ of tumors methylated. However, overall fibrolamellar carcinomas showed low levels of methylation with no differences between fibrolamellar carcinomas and their paired normal livers. However, fibrolamellar carcinomas showed significantly less methylation than hepatocellular carcinomas that arose in the background of viral cirrhosis. Overall, methylation was most strongly linked to viral cirrhosis. In conclusion, fibrolamellar carcinoma shows low levels of methylation. In contrast, higher levels of promoter methylation are associated with hepatocellular carcinomas that arise in the setting of viral induced cirrhosis.
\end{abstract}

Modern Pathology (2008) 21, 670-675; doi:10.1038/modpathol.2008.32; published online 8 February 2008

Keywords: fibrolamellar carcinoma; hepatocellular carcinoma; methylation; viral hepatitis; RASSF1A; HPP1

Fibrolamellar carcinoma is a variant of hepatocellular carcinoma that is unique at the clinical, histological, and molecular levels. It occurs in children and young adults with no evidence for chronic liver disease and is characterized histologically by a unique pattern of intratumoral fibrosis and large, polygonal and eosinophilic cells. The etiology of fibrolamellar carcinoma is unknown. The prognosis for fibrolamellar carcinoma is similar to hepatocellular carcinomas that arise in non-cirrhotic livers, ${ }^{1}$ and both have a better prognosis than hepatocellular carcinomas that arise in cirrhotic livers.

It is generally agreed that hepatocellular carcinoma develops through the accumulation of multiple genetic alterations. In typical hepatocellular

Correspondence: Dr M Torbenson, MD, Department of Pathology, The Johns Hopkins University School of Medicine, 1503 East Jefferson, Room B314, Baltimore, MD 21231, USA.

E-mail: mtorben@jhmi.edu

Received 16 June 2007; accepted 05 September 2007; published online 8 February 2008 carcinoma, chromosomal instability is thought to play an important role, as are mutations in various genes that regulate normal growth and development, apoptosis, and maintenance of genomic integrity. ${ }^{2}$ In addition, epigenetic instability with methylation of tumor suppressor gene promoters has been reported to significantly contribute to the pathogenesis of hepatocellular carcinoma. ${ }^{3}$

Relatively little is known about the molecular biology of fibrolamellar carcinoma, but several studies have demonstrated that, while translocations can be found, fibrolamellar carcinomas are generally chromosomally stable. ${ }^{4-6}$ Likewise, mutations in key genes such as TP53 and CTNNB1 (betacatenin), which are routinely identified in a proportion of typical hepatocellular carcinomas, have not been found in fibrolamellar carcinoma. ${ }^{7-9}$ The limited chromosomal instability and the lack of mutations in key genes found in hepatocellular carcinoma raised the possibility that epigenetic changes, such as methylation of tumor suppressor gene promoters, may play a prominent role in the etiology of fibrolamellar carcinomas. Thus, we 
sought to explore the role of methylation by examining a panel of tumor suppressor gene promoters in fibrolamellar carcinomas and comparing the frequency of methylation to two control groups: hepatocellular carcinomas arising in cirrhotic livers and hepatocellular carcinomas arising in non-cirrhotic livers without evidence for chronic liver disease.

\section{Materials and methods}

\section{Tissues}

After obtaining institutional approval, excess tissues were harvested from surgical pathology specimens and flash frozen in liquid nitrogen and stored at $-80^{\circ} \mathrm{C}$. Routine light microscopy confirmed the histological diagnosis in all cases.

For the fibrolamellar carcinomas, a total of five primary tumors were studied. Of these five cases, four had available paired non-neoplastic liver tissues. We also studied four metastatic deposits of fibrolamellar carcinoma. One of the metastases had a corresponding primary tumor that was also included in this study, while the remaining three had no primary tumor tissues available for analysis. As control groups, we studied the hepatocellular carcinoma tissues of 17 individuals with cirrhosis from chronic hepatitis, including chronic hepatitis C viral infection $(N=10)$ and chronic hepatitis B viral infection $(N=7)$. We also studied individuals who had either cryptogenic cirrhosis or cirrhosis secondary to non-alcoholic fatty liver disease $(N=4)$. Most cases of cryptogenic cirrhosis in the United States are thought be related to burned-out fatty liver disease. As another control group, we also studied individuals with typical hepatocellular carcinoma, but whose background livers were noncirrhotic and who had no identifiable underlying chronic liver disease $(N=10)$. This control group is critical in understanding fibrolamellar carcinoma, as the background livers are normal in both cases and the overall clinical course of fibrolamellar carcinoma is most similar to those hepatocellular carcinomas that arise in non-cirrhotic livers. ${ }^{1}$

\section{Methylation-Specific PCR Amplification}

DNA was extracted from 20 to $25 \mathrm{mg}$ of liver tissues (Dneasy Tissue, Qiagen, Valencia, CA, USA) and DNA bisulfite treatment was performed using EpiTect Bisulfite Kit (Qiagen, Valencia, CA, USA). There is no well-characterized list of tumor suppressor promoters that define a methylator phenotype in hepatocellular carcinoma. Thus, a list of tumor suppressor genes was selected based on a review of the literature and their relevance to hepatocellular carcinoma. Based on the literature and our preliminary data, it seemed unlikely that methylation of any single gene or small number of genes was likely to be informative in differentiating the role of methylation in typical hepatocellular carcinoma vs fibrolamellar carcinoma. Thus, we choose a panel of eight tumor suppressor genes to examine. This was supplemented by the addition of three genes previously shown by our laboratory ${ }^{10}$ to be underexpressed in fibrolamellar carcinomas at the mRNA levels: RARA, HPP1, and FLJ13081. Primers were either selected from the literature (Table 1) or designed using MethPrimer (http:// www.urogene.org/methprimer/).

\section{Statistical Analysis}

The means of three or more groups were compared by ANOVA, while the means of two groups were compared using Student's $t$-tests (Systat version 10).

\section{Results}

\section{Demographic and Histological Findings}

Of the five primary fibrolamellar carcinomas, three were from women and two from men with an age at diagnosis of $29 \pm 14$ years. All individuals with primary tumors underwent surgical resection of their tumor. Patients with metastatic disease underwent resection of these sites with curative intent. All of the cases with metastases were in women, averaging $22 \pm 9$ years at metastatic disease. In all cases, no clinical or histological evidence for background chronic liver disease was seen in the nonneoplastic tissues and the tumors and metastases were histologically typical of fibrolamellar carcinoma. For the viral cirrhosis control group, the average age at diagnosis was $56 \pm 14$ years and included 12 men and 5 women. For the fatty liver or cryptogenic cirrhosis group, the average age at diagnosis was $59 \pm 10$ years and included three men and one woman. For the non-viral, non-cirrhotic control group, the average age at diagnosis was $59 \pm 17$ years and included six men and four women. The ages at resection did not differ significantly between the control groups, $P=0.9$.

\section{Methylation in Fibrolamellar Carcinoma}

Of the three genes previously found to be downregulated in fibrolamellar carcinomas in our laboratory, RARA and FLJ13081 showed low to absent methylation, while HPP1 was methylated in $2 / 5$ $(40 \%)$ of cases (Table 1). Likewise, there was either no or rare methylation of genes involved in cell cycle control, including TP53, P14, P15, and P16. Of all the promoters studied, methylation of the RASSF1 promoter stood out as being the most commonly methylated promoter, with methylation seen in $4 / 5$ cases (Figure 1). E-cadherin was partially methylated in all fibrolamellar carcinomas but the paired non-neoplastic tissues were also partially 


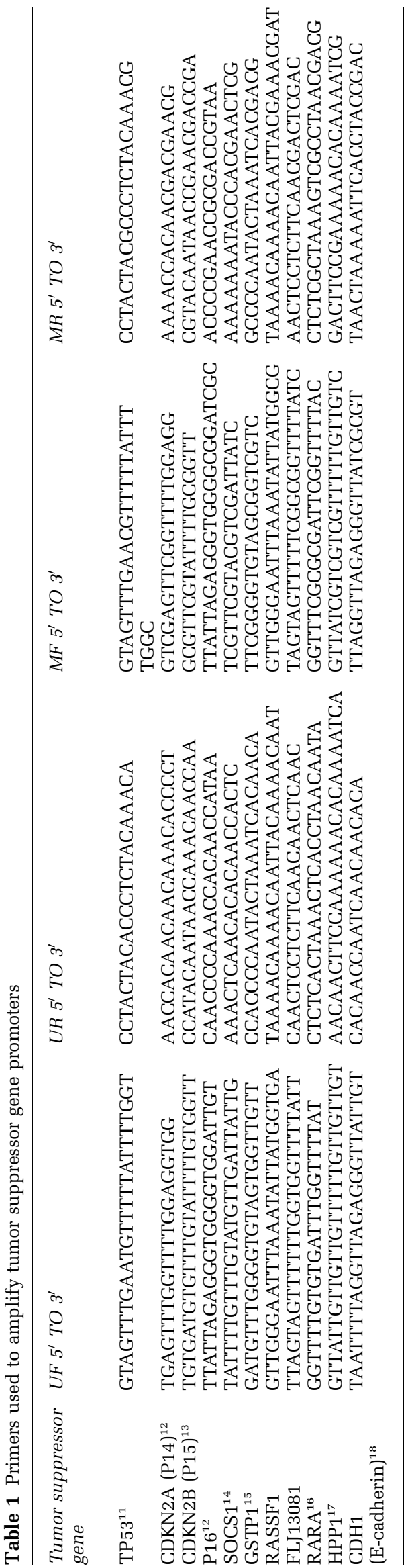

methylated in all cases, suggesting that methylation of this gene was not qualitatively different in the tumor vs non-tumor. Overall, the number of methylated genes in fibrolamellar carcinoma did not differ significantly from their paired normal tissues, $P=0.39$.

\section{Methylation in FLC Metastases}

Metastatic deposits of fibrolamellar carcinoma have dramatically increased numbers of over- and underexpressed genes compared to the primary tumors. ${ }^{10}$ Interestingly, this change in the expression levels of numerous genes does not appear to be associated with increased numbers of methylated genes, $P=0.43$ (Figure 2).

\section{Methylation in Control Groups}

Within the group of hepatocellular carcinomas that arose in the setting of cirrhosis, examination of the data (Tables 2 and 3) showed that cirrhotic livers in the setting of cryptogenic liver disease and non-alcoholic fatty liver disease had significantly lower levels of methylation than virus-associated cirrhosis, $P=0.014$, and for subsequent analysis, the cirrhotics were studied as two separate groups: viral cirrhosis and non-viral cirrhosis.

The number of methylated promoters in fibrolamellar carcinomas did not differ significantly with those hepatocellular carcinomas that arose in noncirrhotic livers, $P=0.26$, as well as hepatocellular carcinomas that arose in the setting of non-viral cirrhosis, $P=0.87$. However, fibrolamellar carcinomas had significantly lower levels of methylation than hepatocellular carcinomas that arose in the setting of viral induced cirrhosis, $P=0.023$. In fact, hepatocellular carcinomas that arose in the setting of viral-induced cirrhosis had higher levels of promoter methylation than all other groups (Figure 2).

\section{Discussion}

This study is the first to examine the methylation patterns of tumor suppressor gene promoters in fibrolamellar carcinoma. While a formal definition of a methylator phenotype has not been well defined at this time for hepatocellular carcinomas, fibrolamellar carcinomas do not appear to have a 'methylator phenotype'. The results from this study show that fibrolamellar carcinomas have lower levels of promoter methylation than hepatocellular carcinomas that arise in the setting of viral cirrhosis and that overall the levels of methylation in fibrolamellar carcinomas are similar to those hepatocellular carcinomas that arise in non-cirrhotic livers. While the number of cases of cryptogenic cirrhotics and cirrhosis due to fatty liver disease was small, this 
Table 2 Methylation of tumor suppressor genes in fibrolamellar carcinoma

\begin{tabular}{|c|c|c|c|}
\hline Tumor suppressor gene & Normal livers $^{\mathrm{a}}(\mathrm{N}=4)$ & $F L C$, primary $(\mathrm{N}=5)$ & $F L C$, metastasis $(\mathrm{N}=4)$ \\
\hline CDH1 (E-cadherin) & $4(100 \%)$ & $5(100 \%)$ & $3(75 \%)$ \\
\hline RASSF1 & $0(0 \%)$ & $4(80 \%)$ & $2(50 \%)$ \\
\hline CDKN2B (P15) & $1(25 \%)$ & $2(40 \%)$ & $0(0 \%)$ \\
\hline HPP1 & $1(25 \%)$ & $2(40 \%)$ & $1(25 \%)$ \\
\hline CDKN2A (P14) & $1(25 \%)$ & $2(40 \%)$ & $1(25 \%)$ \\
\hline GSTP1 & $1(25 \%)$ & $1(20 \%)$ & $0(0 \%)$ \\
\hline P16 & $1(25 \%)$ & $1(20 \%)$ & $0(0 \%)$ \\
\hline RARA & $0(0 \%)$ & $1(20 \%)$ & $1(25 \%)$ \\
\hline FLJ13081 & $1(25 \%)$ & $0(0 \%)$ & $1(25 \%)$ \\
\hline SOCS1 & $2(50 \%)$ & $0(0 \%)$ & $0(0 \%)$ \\
\hline TP53 & $0(0 \%)$ & $0(0 \%)$ & $0(0 \%)$ \\
\hline
\end{tabular}

${ }^{\mathrm{a}}$ Four of the five FLC had paired normal liver available for study.

Table 3 Methylation of tumor suppressor genes in primary fibrolamellar carcinoma and hepatocellular carcinomas

\begin{tabular}{|c|c|c|c|c|}
\hline Tumor suppressor gene & $\begin{array}{l}\text { HCC, viral cirrhosis } \\
(\mathrm{N}=17)\end{array}$ & $\begin{array}{l}\text { HCC non-viral cirrhosis } \\
\qquad(\mathrm{N}=4)\end{array}$ & $\begin{array}{l}\text { HCC, non-cirrhotic } \\
(\mathrm{N}=10)\end{array}$ & $\begin{array}{l}\text { FLC, primary } \\
(\mathrm{N}=5)\end{array}$ \\
\hline RASSF1 & $15(88 \%)$ & $2(50 \%)$ & $5(50 \%)$ & $4(80 \%)$ \\
\hline CDH1 (E-cadherin) & $15(88 \%)$ & $2(50 \%)$ & $6(60 \%)$ & $5(100 \%)$ \\
\hline GSTP1 & $13(76 \%)$ & $3(75 \%)$ & $2(20 \%)$ & $1(20 \%)$ \\
\hline P16 & $11(65 \%)$ & $3(75 \%)$ & $2(20 \%)$ & $1(20 \%)$ \\
\hline SOCS1 & $11(65 \%)$ & $0(0 \%)$ & $2(20 \%)$ & $0(0 \%)$ \\
\hline FLJ13081 & $10(59 \%)$ & $1(25 \%)$ & $3(30 \%)$ & $0(0 \%)$ \\
\hline CDKN2B (P15) & $9(53 \%)$ & $1(25 \%)$ & $2(20 \%)$ & $2(40 \%)$ \\
\hline HPP1 & $8(47 \%)$ & $1(25 \%)$ & $2(20 \%)$ & $2(40 \%)$ \\
\hline RARA & $3(18 \%)$ & $0(0 \%)$ & $0(0 \%)$ & $1(20 \%)$ \\
\hline CDKN2A (P14) & $2(12 \%)$ & $0(0 \%)$ & $0(0 \%)$ & $2(40 \%)$ \\
\hline TP53 & $0(0 \%)$ & $0(0 \%)$ & $0(0 \%)$ & $0(0 \%)$ \\
\hline
\end{tabular}

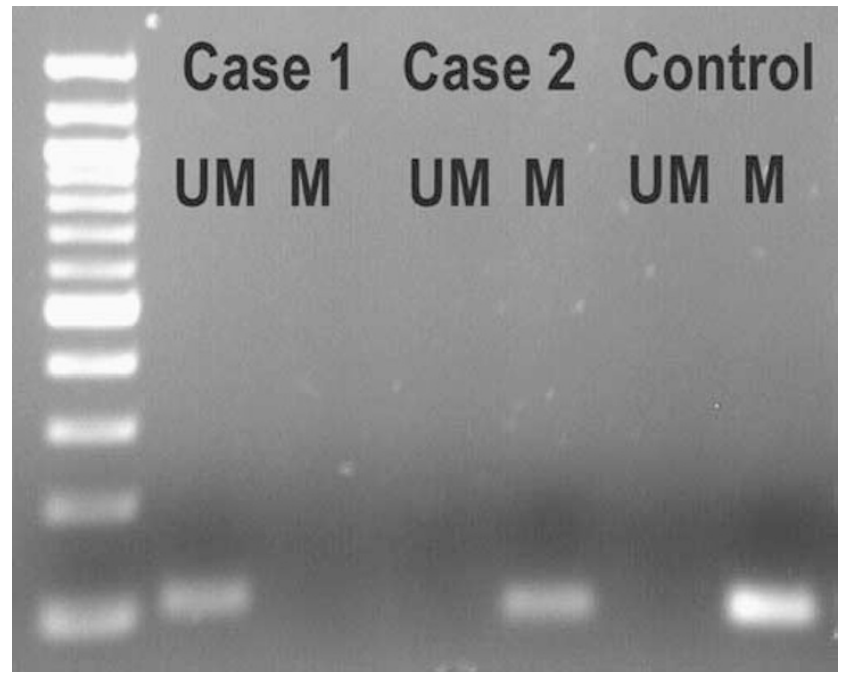

Figure 1 A representative example of methylation results for RASSF1A. Case 1 shows methylation in a typical HCC; case 2 shows methylation on a FLC. Hep3B DNA was used as a positive control.

group appears to more closely resemble those hepatocellular carcinomas that arise in non-cirrhotic livers. Together, these data support a paradigm where viral hepatitis-induced cirrhosis gives rise

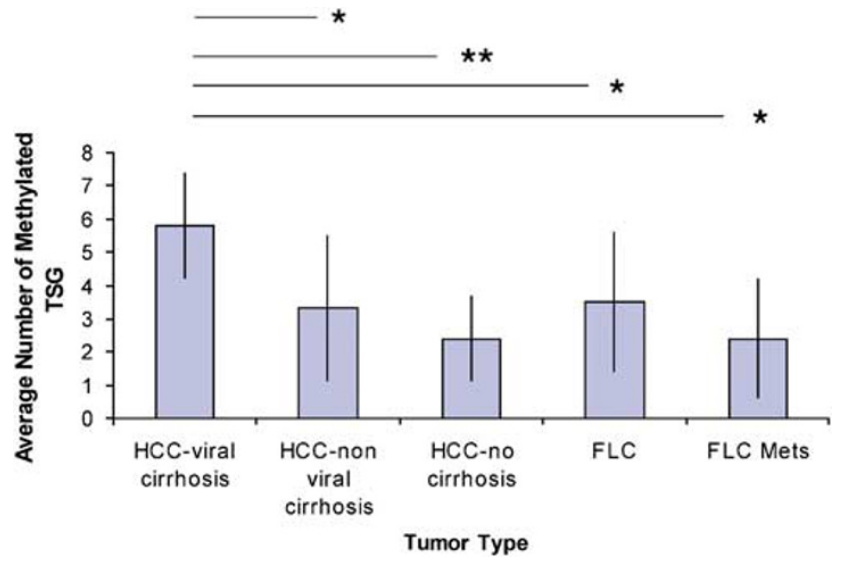

Figure 2 The average number of methylated tumor suppressor gene promoters varies in association with underlying status of the background liver. The first column shows hepatocellular carcinomas associated with viral cirrhosis, the second column shows hepatocellular carcinoma arising in non-cirrhotic livers with no known underlying liver disease, while the third column shows primary fibrolamellar carcinomas and the fourth column metastatic fibrolamellar carcinoma. Differences that were statistically significant are shown by asterisks, ${ }^{*} P<0.05$; ${ }^{*} P<0.001$.

to tumors with higher levels of tumor suppressor gene methylation, the 'methylator phenotype', whereas hepatocellular carcinomas and fibrolamellar carcinomas that arise in non-cirrhotic livers give 
rise to cancers where methylation is less prominent. This model is supported by a previous study that also found a link between viral hepatitis B and the methylator phenotype. ${ }^{15}$ The mechanism for this link between viral cirrhosis and the methylator phenotype is not well defined, but it is of interest to note that hepatitis B can directly upregulate DNA methyltransferase type $1 .{ }^{19}$ At the individual tumor suppressor gene level, others have also observed a higher frequency of methylation in hepatocellular carcinomas that arise in the setting of cirrhosis compared to those in noncirrhotic livers for RASSF1A, CIS, and SOCS1. ${ }^{20}$ Additional cases of non-viral cirrhosis, such as cryptogenic cirrhosis, non-alcoholic fatty liver cirrhosis, hereditary hemochromatosis, and so on, will need to be examined to more fully define the levels of methylation in this subgroup of hepatocellular carcinomas, but the findings in this study suggest that cirrhosis per se is not as strongly associated with a methylator phenotype as viral hepatitis induced cirrhosis.

Overall, RASSF1A and e-cadherin were the two genes most commonly methylated in fibrolamellar carcinomas. None of these are unique to fibrolamellar carcinoma and are in fact commonly methylated in hepatocellular carcinomas of the usual sort in both this and other studies. Two of these promoters, $C D H 1$ and $S F N$, were also found to be partially methylated in the paired normal livers of cases with fibrolamellar carcinoma. In contrast, RASSF1A was methylated only in the fibrolamellar carcinomas and not in the paired non-neoplastic liver tissues. RASSF1 encodes for a protein with a number of disparate functions, from regulating microtubule assembly to regulation of apoptosis and the cell cycle. ${ }^{21}$ RASSF1A promoter methylation has been reported in numerous types of cancer including hepatocellular carcinomas, where typically more than $80 \%$ of hepatocellular carcinomas show methylation. ${ }^{13,22,23}$ Thus, based on this study and others reported in the literature, RASSF1A appears to be methylated in all known primary liver tumors that show hepatocellular differentiation, including hepatoblastomas, ${ }^{24}$ hepatic adenomas, ${ }^{25}$ fibrolamellar carcinomas, and hepatocellular carcinoma. ${ }^{13,22,23}$

This study is also the first to show a role for methylation of HPP1 in primary liver tumors. HPP1 has been previously reported to be methylated in adenocarcinomas from the esophagus, stomach, small bowel and colorectum. ${ }^{26-31}$ A previous study found HPP1 to be downregulated in fibrolamellar carcinomas at the mRNA level ${ }^{10}$ and the current results demonstrate that the downregulation of HPP1 expression is associated with promoter methylation. The function of this gene is unknown; however, it codes for a transmembrane protein that contains both epidermal growth factor and follistatin-like domains and may play a role in inhibiting serine proteases.
Further studies will be required to understand the significance of methylation in non-neoplastic liver tissues of individuals with fibrolamellar carcinomas. One possibility is that these changes are preneoplastic. For example, if one gene copy is inactivated by methylation, then an additional hit from point mutations or deletions may lead to the loss of protein expression. However, given that these changes were observed in histologically normal livers with no morphological features to suggest pre-neoplastic changes, their significance at this time remains unclear. Furthermore, methylation of promoters may be age related in some cases. For example, both e-cadherin and RASSF1A methylation has been reported to increase with age in normal liver tissues, ${ }^{25,32}$ although the young ages of individuals with fibrolamellar carcinoma suggest that this explanation may not account for our findings.

The low levels of promoter methylation in fibrolamellar carcinomas as well as hepatocellular carcinomas that arise in non-cirrhotic livers are a critical observation that is likely to be important in designing future chemotherapeutic regiments for hepatocellular carcinoma. Demethylating agents that can restore the function of inactivated tumor suppressor genes are an attractive target for new therapeutics for hepatocellular carcinomas. ${ }^{33}$ Our findings suggest that they may be applicable to the majority of hepatocellular carcinomas, since approximately $80 \%$ arise in the background of viral hepatitis-induced liver cirrhosis. However, our findings also suggest that different treatment strategies may be needed for those primary hepatocellular carcinomas and fibrolamellar carcinomas that arise in non-cirrhotic livers and do not show a 'methylator phenotype'.

Because of the rarity of fibrolamellar carcinomas, this study is limited in part by the relatively few samples that were studied. However, the morphological and genetic homogeneity of this tumor strongly suggests that these findings are broadly applicable to fibrolamellar carcinomas in general. The findings in this study are further strengthened by our ability to use as a control group hepatocellular carcinomas that arose in non-cirrhotic livers. This group of hepatocellular carcinomas represents another uncommon subset of tumors that remains poorly understood at the etiological and molecular levels, but is a critical control group for interpreting the data on fibrolamellar carcinomas.

In conclusion, fibrolamellar carcinomas overall do not show a 'methylator phenotype'. In fact, the strongest association between increased methylation of tumor suppressor gene promoters was seen with viral-induced cirrhosis. Of the genes studied, RASSF1A was the most commonly methylated gene in fibrolamellar carcinomas and was seen in $80 \%$ of the primary tumors and $50 \%$ of metastases. 


\section{References}

1 Kakar S, Burgart LJ, Batts KP, et al. Clinicopathologic features and survival in fibrolamellar carcinoma: comparison with conventional hepatocellular carcinoma with and without cirrhosis. Mod Pathol 2005; 18:1417-1423.

2 Farazi PA, DePinho RA. Hepatocellular carcinoma pathogenesis: from genes to environment. Nat Rev Cancer 2006;6:674-687.

3 Herath NI, Leggett BA, MacDonald GA. Review of genetic and epigenetic alterations in hepatocarcinogenesis. J Gastroenterol Hepatol 2006;21:15-21.

4 Marchio A, Pineau $\mathrm{P}$, Meddeb $\mathrm{M}$, et al. Distinct chromosomal abnormality pattern in primary liver cancer of non-B, non-C patients. Oncogene 2000; 19:3733-3738.

5 Terracciano LM, Tornillo L, Avoledo P, et al. Fibrolamellar hepatocellular carcinoma occurring 5 years after hepatocellular adenoma in a 14-year-old girl: a case report with comparative genomic hybridization analysis. Arch Pathol Lab Med 2004;128:222-226.

6 Wilkens L, Bredt M, Flemming P, et al. Cytogenetic aberrations in primary and recurrent fibrolamellar hepatocellular carcinoma detected by comparative genomic hybridization. Am J Clin Pathol 2000;114: 867-874.

7 Kannangai R, Wang J, Liu QZ, et al. Survivin overexpression in hepatocellular carcinoma is associated with p53 dysregulation. Int J Gastrointest Cancer 2005;35:53-60.

8 Honda K, Sbisa E, Tullo A, et al. p53 mutation is a poor prognostic indicator for survival in patients with hepatocellular carcinoma undergoing surgical tumour ablation. Br J Cancer 1998;77:776-782.

9 Terris B, Pineau P, Bregeaud L, et al. Close correlation between beta-catenin gene alterations and nuclear accumulation of the protein in human hepatocellular carcinomas. Oncogene 1999;18:6583-6588.

10 Kannangai R, Vivekanandan P, Martinez-Murillo F, et al. Fibrolamellar carcinomas show over expression of genes in the RAS, MAPK, PIK3, and xenobiotic degradation pathways. Hum Pathol 2007;38:639-644.

11 Park HJ, Yu E, Shim YH. DNA methyltransferase expression and DNA hypermethylation in human hepatocellular carcinoma. Cancer Lett 2006;233: 271-278.

$12 \mathrm{Yu}$ J, Zhang H, Gu J, et al. Methylation profiles of thirty four promoter-CpG islands and concordant methylation behaviours of sixteen genes that may contribute to carcinogenesis of astrocytoma. BMC Cancer 2004;4:65.

$13 \mathrm{Yu}$ J, Ni M, Xu J, et al. Methylation profiling of twenty promoter-CpG islands of genes which may contribute to hepatocellular carcinogenesis. BMC Cancer 2002; $2: 29$.

14 Fukushima N, Sato N, Sahin F, et al. Aberrant methylation of suppressor of cytokine signalling-1 (SOCS-1) gene in pancreatic ductal neoplasms. Br J Cancer 2003;89:338-343.

15 Katoh H, Shibata T, Kokubu A, et al. Epigenetic instability and chromosomal instability in hepatocellular carcinoma. Am J Pathol 2006;168:1375-1384.

16 Chim CS, Wong SY, Pang A, et al. Aberrant promoter methylation of the retinoic acid receptor alpha gene in acute promyelocytic leukemia. Leukemia 2005;19: 2241-2246.
17 Sato F, Shibata D, Harpaz N, et al. Aberrant methylation of the HPP1 gene in ulcerative colitisassociated colorectal carcinoma. Cancer Res 2002;62: 6820-6822.

18 Ueki T, Toyota M, Sohn T, et al. Hypermethylation of multiple genes in pancreatic adenocarcinoma. Cancer Res 2000;60:1835-1839.

19 Lee JO, Kwun HJ, Jung JK, et al. Hepatitis B virus X protein represses E-cadherin expression via activation of DNA methyltransferase 1. Oncogene 2005;24: 6617-6625.

20 Calvisi DF, Ladu S, Gorden A, et al. Ubiquitous activation of Ras and Jak/Stat pathways in human HCC. Gastroenterology 2006;130:1117-1128.

21 Agathanggelou A, Cooper WN, Latif F. Role of the Rasassociation domain family 1 tumor suppressor gene in human cancers. Cancer Res 2005;65:3497-3508.

22 Zhang YJ, Ahsan H, Chen Y, et al. High frequency of promoter hypermethylation of RASSF1A and p16 and its relationship to aflatoxin B1-DNA adduct levels in human hepatocellular carcinoma. Mol Carcinog 2002; 35:85-92.

23 Zhong S, Yeo W, Tang MW, et al. Intensive hypermethylation of the $\mathrm{CpG}$ island of Ras association domain family $1 \mathrm{~A}$ in hepatitis $\mathrm{B}$ virus-associated hepatocellular carcinomas. Clin Cancer Res 2003;9: 3376-3382.

24 Sugawara W, Haruta M, Sasaki F, et al. Promoter hypermethylation of the RASSF1A gene predicts the poor outcome of patients with hepatoblastoma. Pediatr Blood Cancer 2006;49:240-249.

25 Di Gioia S, Bianchi P, Destro A, et al. Quantitative evaluation of RASSF1A methylation in the nonlesional, regenerative and neoplastic liver. BMC Cancer 2006;6:89.

26 Hamilton JP, Sato F, Greenwald BD, et al. Promoter methylation and response to chemotherapy and radiation in esophageal cancer. Clin Gastroenterol Hepatol 2006;4:701-708.

27 Brucher BL, Geddert H, Langner C, et al. Hypermethylation of hMLH1, HPP1, p14(ARF), p16(INK4A) and APC in primary adenocarcinomas of the small bowel. Int J Cancer 2006;119:1298-1302.

28 Ebert MP, Mooney SH, Tonnes-Priddy L, et al. Hypermethylation of the TPEF/HPP1 gene in primary and metastatic colorectal cancers. Neoplasia 2005;7: 771-778

29 Suzuki M, Shigematsu H, Shames DS, et al. DNA methylation-associated inactivation of TGFbeta-related genes DRM/Gremlin, RUNX3, and HPP1 in human cancers. Br J Cancer 2005;93:1029-1037.

30 Shibata DM, Sato F, Mori Y, et al. Hypermethylation of HPP1 is associated with hMLH1 hypermethylation in gastric adenocarcinomas. Cancer Res 2002;62: 5637-5640.

31 Young J, Biden KG, Simms LA, et al. HPP1: a transmembrane protein-encoding gene commonly methylated in colorectal polyps and cancers. Proc Natl Acad Sci USA 2001;98:265-270.

32 Waki T, Tamura G, Sato M, et al. Age-related methylation of tumor suppressor and tumor-related genes: an analysis of autopsy samples. Oncogene 2003;22: 4128-4133.

33 Roberts LR, Gores GJ. Emerging drugs for hepatocellular carcinoma. Expert Opin Emerg Drugs 2006;11: 469-487. 